\title{
Evaluación de la factibilidad de un sistema de entrenamiento combinado en el desarrollo de la fuerza explosiva de los miembros inferiores de los taekwondocas Assessment of the feasibility of a combined training system in the development of explosive strength of the lower limbs of taekwondocas \\ *Lisbet Guillen Pereira, ${ }^{* *}$ Angel Freddy Rodriguez Torres, ${ }^{* *}$ Giovanny Capote Lavandero, **Pablo Anthony Rendón

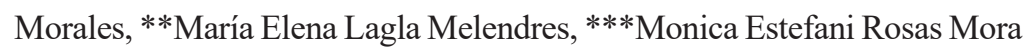 \\ *Universidad Metropolitana del Ecuador (Ecuador), **Universidad Central del Ecuador (Ecuador), ***Pontificia Universidad Católica del Ecuador (Ecuador)
}

Resumen: La investigación se centró en evaluar el impacto de un sistema de entrenamiento combinado para desarrollar la fuerza explosiva de los miembros inferiores de los taekwondocas, viene a reforzar el enfoque del desarrollo de la capacidad mediante una lógica metodológica que parte de la creación de las condiciones morfológicas y funcionales para asegurar la potenciación de una hipertrofia sarcoplasmática, como base del desarrollo de la hipertrofia sarcomérica y finalmente favorecer la conversión a potencia y el desarrollo de la fuerza explosiva, en concordancia se transitó por un diagnóstico, elaboración y validación, utilizando convenientemente métodos y técnicas como: entrevista, encuesta, observación, la prueba, la medición, el criterios de experto, la estadística descriptiva e inferencial y el pre-experimento, este último con tres momentos (pre-test/ intervención de 16 semanas/ post-test), participaron 20 taekwondocas universitarios Categoría Sénior y dos entrenadores. Se consideró la evaluación de la fuerza máxima (1RM) de los planos musculares que intervienen directamente en las técnicas de pateo, por estar asociada con la calidad del reclutamiento de la fibras motoras; la evaluación de la fuerza explosiva se realizó mediante el test de salto vertical: Squat Jump (SJ) extraído del protocolo del test de Bosco, para ello fue necesario emplear una plataforma de contacto digital. Los datos se compararon mediante una prueba $\mathrm{T}$ de diferencias de medias, cuyos resultados mostraron cambios significativos $(p=.000)$ entre el pre y post tratamiento, verificándose Hi, concluyendo que el sistema de entrenamiento combinado mejoró el desarrollo de la fuerza explosiva de los miembros inferiores de los taekwondocas.

Palabras claves: Taekwondo, Fuerza explosiva, Técnicas de pateo, Sistema de entrenamiento, Sistema de ejercicios.

Summary: The research focused on assessing the impact of a combined training system to develop the explosive strength of the lower limbs of the taekwondoins. It wants to reinforce the approach of capacity development through a methodological logic that starts from the creation of morphological and functional conditions to ensure the enhancement of a sarcoplasmic hypertrophy, as a basis for the development of sarcomeric hypertrophy, and finally, favor the conversion to power and the development of the explosive force. At the same time, it went through a diagnosis, elaboration and data validation, using propper methods and techniques such as: interview, survey, observation, testing, measurement, expert criteria, descriptive and inferential statistics, and the pre-experiment. The last one with three stages (pre-test / 16-week intervention / post-test) Twenty senior category university taekwondoins and two coaches participated. The evaluation of the maximum force (1RM) of the muscular planes that directly influence in the kicking techniques was considered. As it was associated to the quality of motor fiber recruitment; The evaluation of the explosive force was performed using the vertical jump test: Squat Jump (SJ) extracted from the Bosco test protocol. For this, it was necessary to use a digital contact platform. The data were compared using a T-test, the results of which showed significant changes $(p=.000)$ between the pre and post treatment, verifying Hi. Concluding that the combined training system improved the development of the explosive strength of the limbs lower of the taekwondoinst. Keywords: Taekwondo, Explosive strength, Kicking techniques, Training system, Exercise system.

\section{Introducción}

El taekwondo es un arte marcial que clasifica como deporte táctico, de oposición y de enfrentamiento directo, las acciones suelen ser aciclicas, variadas y muy rápidas; desde el punto de vista técnico se evidencia un predomino del empleo de las técnicas de pateo en el trabajo de oposición, mostrando una frecuencia entre 30 y 40 pateos durante el combate y entre $10 \mathrm{a} 16$ por asaltos, de igual manera se ejecutan combinaciones que pueden llegar a oscilar entre tres y cinco ejecuciones por minuto (Kazemi, Waalen, Morgan \& White, 2006; González, 2011; González, Iglesias, Mirallas \& Esparza, 2011; Núñez, 2011; Bridge, Ferreira, Chaabene, Pieter \& Franchini, 2014).

En relación al tema y desde el punto de vista capacitivo Martínez, Pegueros, Ortiz, del Villar, Flores \& Pineda (2014) corroboran mediante una investigación que la fuerza y el

Fecha recepción: 29-05-20. Fecha de aceptación: 18-07-20

Lisbet Guillen Pereira

guillenp7212@gmail.com balance muscular de las extremidades inferiores constituyen atributos importantes en el Taekwondo, asumen que los altos niveles de fuerza en la musculatura extensora y flexora de los miembros inferiores representa una particularidad que asegura una certera y eficaz ejecución de las combinaciones, requeridas para la ejecución de la técnica de pateo, los saltos, los giros y los desplazamientos, de lo cual se infiere el necesario desarrollo de la fuerza y sus diferentes manifestaciones para garantizar el rendimiento deseado.

En este punto la proyección dinámica del desarrollo de las capacidades, a partir del análisis de las especificidades de la disciplina, apuntan a considerar la resistencia lactácida o de corta duración, la potencia anaeróbica y la fuerza explosiva como capacidades determinantes para la ejecución de las técnicas de pateo y otras técnicas complementarias. Considerado el enfoque de la investigación vale clarificar que la Fuerza explosiva es entendida como la capacidad de realizar una acción con el máximo de fuerza aplicada en el mínimo tiempo posible (Bompa, 1999; Carazo, 2013; Cardozo, VeraRivera, Conde-Cabezas \& Yánez, 2017), su desarrollo máximo, para las técnicas ejecutadas de los miembros inferiores, 
dependerá de la composición muscular del individuo, del impulso, de la sincronización de la cadena biocinemática que intervine en la acción motora, de la coordinación intra e interrmuscular y de la Fuerza máxima de los planos musculares implicados en la mecánica de la acción (González-Badillo \& Gorostiaga, 1995; León, Ortiz, Lara, Marcillo \& Guerrero, 2017)

Diversos autores coinciden en plantear que para que un movimiento pueda ser calificado de potente deben darse dos condiciones fundamentales: que el movimiento venza grandes resistencias y que alcance grandes aceleraciones, por tanto, en la intención debe valorase la calidad de la fuerza muscular explosiva y la fuerza de partida promovida durante la iniciación de la ejecución (Álvarez-Bedolla, 2003a; Álvarez-Bedolla, 2003b; Torrealba-Paéz, Torrealba-Paéz, Bolivar-Monaterio, Pacífico-Casadiego \& Núñez-Rodríguez, 2015; Briñones, Camejo \& Rosales, 2017; Méndez, 2018).

Es por ello que al direccionar de forma correcta la preparación física en el Taekwondo, es necesario manejar, las especificidades del deporte desde el punto de vista técnicotáctico y el carácter de los esfuerzos en el desempeño competitivo, lo que induce hacia el análisis estructural funcional de las acciones, tomando en cuenta la musculatura implicada, la duración mínima de los combates ( 6 minutos en adultos y 2 minutos por asalto), las especificidades de las acciones ante la variabilidad de su ejecución y las características del entorno de formación (Morales, 2014b; Medina-Maes, 2015; Morales, 2015, Guillen, Ale \& Coral, 2017; León et al., 2017; Norjali-Wazir, Van-Hiel, Mostaert, Deconinck, Pion \& Lenoir, 2019).

En relación al tema, diversos estudios (Morales, 2014a; James, Haff, Kelly \& Beckman, 2016; Guillén et al., 2017; Prieto, Sagat, Ben-Brahim \& Sedlacek, 2020) corroboran que el desarrollo del proceso de preparación física de los taekwondocas, regularmente, se ve afectado por las limitaciones en el enfoque de los sistemas de entrenamiento utilizados para desplazar, de forma coherente y en el marco del periodo de preparación, los diferentes tipos de fuerza por etapas, por tanto se caracterizan por ser estáticos, con alto componente empírico y no ajustados en su base a las exigencias actuales que se derivan del sistema de competición, lo que se justifica frente al desconocimiento de las bases biológicas del ejercicio físico, los efectos fisiológicos de la carga y los modos de evaluación que podrían respaldar la concepción y despliegue de dicho proceso.

Múltiples han sido las alternativas de solución para direccionar el desarrollo de la fuerza y el manejo de la evaluación de la capacidad en los deportes tácticos, en los que se incluyen el Taekwondo. La revisión teórica realizada para el presente estudio destaca los aportes de Nikolaidis, Chtourou, Torres-Luque, Tasiopoulos, Heller \& Padulo (2015) los cuales demuestran cambios significativos en los atributos físicos de los taekwondistas, las características fisiológicas y las respuestas adaptativas como resultado de la implementación de un sistema de entrenamiento que favorece el desarrollo de la potencia y de la fuerza-velocidad, para cuya evaluación emplearon el test de salto vertical y la medición de la capacidad mediante ergómetro.

García-Asencio, Sánchez-Moreno \& González-Badillo (2016), proponen para mejorar el rendimiento en el salto ver- tical de voleibolistas un entrenamiento combinado, en el cual se articulan ejercicio de fuerza y saltos, de igual manera Sánchez-Sixto \& Floría (2017) validan un entrenamiento combinado donde utilizan ejercicios de fuerza, velocidad y desplazamiento para mejorar el salto en basketbolistas.

Obras de especial relevancia en el marco del Taekwondo (Chiodo, Tessitore, Lupo, Ammendolia, Cortis \& Capranica, 2012; Cheng, Wang, Kuo, Wang \& Huang, 2015; Preuschl, Hassmann \& Baca, 2016; Rendón et al., 2017; Sánchez-Sixto \& Floría, 2017) justifican la pertinencia de sistemas de entrenamiento centrados en el desarrollo de la fuerza explosiva para favorecer el perfeccionamiento del empleo de las técnicas de pateo en el trabajo de oposición, considerando según la etapa de preparación, la utilización de ejercicios con cargas de peso ligeras pero ejecutadas a máxima velocidad (Cometti, 2002; Kim, Stebbins, Chai \& Song, 2011), entrenamiento con cargas progresivas (González-Badillo \& Izquierdo-Redín, 2006), entrenamientos propioceptivos y de desarrollo de la fuerza muscular (Sihyun, Sang-Kyoon, Sukhoon, Ji, Sung \& Jiseon, 2018), ejercicios para el desarrollo de la fuerza máxima, ejercicios pliométricos, ejercicios utilizando medios específicos y auxiliares (León et al., 2017), así como entrenamiento de intervalos de alta intensidad (Monks, Seo, Kim, Jung \& Song, 2017).

Dentro de los modos de evaluación de la fuerza explosiva en Taekwondocas Cardozo \& Moreno-Jiménez (2018), destacan múltiples procedimientos empleados para la evaluación de la capacidad como resultado de una profunda revisión teórica, dentro de estos recalcan algunos test de saltos ajustados al protocolo de Bosco (Bosco, 1994): test de salto Squat Jump o salto sin contra-movimiento (SJ), test de salto Countermovement Jump o salto con contramovimiento (CMJ), test de salto Countermovement Jump with Arm Swing o salto con contramovimiento, test de Abalakov (CMJA) o también conocido como balanceo de brazos, test de salto Standing Long Jump o salto largo a pie junto con balanceo de brazos (LJ), el test de salto Vertical Jump Test o test de saltar y alcanzar (VJ), y el test de tres saltos o 3-Hop Jump Test (3HJ), vale aclarar, que el estudio indica que los procedimiento empleados consignan entre dos y tres intentos, registrando el mejor resultado.

Los medios auxiliares empleados para la evaluación de la Fuerza Explosiva han variado, según la época y el contexto, en el $100 \%$ de los casos verificados utilizan como unidad de medida el cm; dentro de las herramientas empleadas se encuentran los dispositivos de salto vertical ajustables, medidor digital con un sistema de citan métrica de retroceso y soporte ajustable a la cintura, sistema de obtención óptica de datos con una barra trasmisora y receptora, plataforma de fuerza portátil con sensores piezoeléctricos de alta precisión, plataforma de contacto con temporizador digital, plataforma de contacto con cronometro de alta resolución, sistema de captación de datos con un encorder óptico y un soporte ajustable para la cintura que mide la exactitud posicional y el ángulo de elevación (García-Orea, Heredia, Aguilera, Arenas-Dalla \& Pérez-Caballero, 2017; Cardozo \& MorenoJiménez, 2018; Ojeda-Aravena, Azócar-Gallardo, HérnandezMosqueira, Herrera-\& Valenzuela, 2021).

Considerando los elementos antes expuesto la presente investigación se centró en: evaluar la factibilidad de un sis- 
tema de entrenamiento combinado para desarrollar la fuerza explosiva de los miembros inferiores de los taekwondocas objeto de estudio.

\section{Metodología}

\section{Población y muestra}

Para desarrollar el estudio se empleó dos unidades de análisis compuestas por dos entrenadores de Taekwondo (informantes claves) y 20 atletas universitarios de la disciplina de la categoría sénior, seleccionado mediante diseño no probabilístico intencional a conveniencia de los investigadores, asumiendo como criterio de intencionalidad que los atletas se encuentren formando parte del equipo regular del club, estos representan el $71.42 \%$ de la población (28 taekwondocas), y poseen un promedio de edad de $22 \pm 2.8$ años, con una experiencia en la práctica de esta Arte Marcial de \pm 4 años, la estatura promedio de la muestra es de $1.62 \pm$ $0.11 \mathrm{~m}$, la media de la estatura del sexo femenino (8 atletas) es de 1,53 $\pm 0,9 \mathrm{~m}$ y en el sexo masculino (12 atletas) la estatura promedio es de $1.67 \pm 0.9 \mathrm{~m}$, el peso del sexo femenino osciló entre 44 y $75 \mathrm{~kg}$ mostrando una media de $60.62 \pm 11.84 \mathrm{~kg}$, y en el sexo masculino el peso fue desde los 54 hasta los $98 \mathrm{~kg}$ con una media de $71.16 \pm 12.69 \mathrm{~kg}$. La muestra intervino en la fase diagnóstica y en la fase de validación empírica del estudio.

Para la evaluación de los instrumentos empleados y la calidad formal de la propuesta se seleccionaron 15 expertos de una bolsa inicial de 38, cifra que se encuentra dentro de las recomendadas por Zatsiorsky (1989) y Crespo (2007). Para la conformación de la bolsa inicial se tuvo en consideración diferentes criterios enmarcados en: competencia, dominio del tema, disposición a participar, tiempo real para hacer el análisis, efectividad de su actividad profesional, datos que se obtuvieron mediante una entrevista personal.

\section{Material y método}

Para este estudio se asumió una investigación experimental, ya que se enfocó en determinar el impacto de un sistema de entrenamiento combinado sobre el objeto de estudio (fuerza explosiva), para lo cual se compararon dos momentos dados de la realidad. En concordancia se transitó por tres fases: diagnóstico, elaboración y validación de la propuesta.

En este orden el diseño empleado para alcanzar los objetivos de la investigación, fue congruente con los procesos de construcción del conocimiento, empleando coherentemente métodos del nivel teórico, empírico y estadístico matemático y técnicas para la recolección y procesamiento de la información: dentro de los que se destaca la entrevista, la encuesta y la observación, cuyos instrumentos fueron validados a través del criterio de expertos y del Coeficiente alfa de Cronbach, para lo cual se implementó una encuesta piloto, mostrando resultados mayores a 0.7 (rango: e» $8.6 \mathrm{x}$ d» 9.7) en cada instrumento (Celina \& Campo, 2005), se comprobó por tanto que los indicadores declarados en cada instrumentos son aquellos que mayor información ofrecen de la variable relevante.

De igual forma se utilizó la prueba (3):

1. Prueba técnica para realizar un análisis estructural funcional de las acciones con el objetivo de identificar, en la fase diagnóstica, los planos musculares que inciden fundamentalmente en la ejecución de los pateos, giros y desplazamientos

2. Prueba de Fuerza Máxima (1RM)

3. Prueba de Salto vertical o Squat Jump (SJ) utilizando una plataforma digital para medir la fuerza explosiva, la cual contempló tres intentos permitiendo elegir el mejor resultado.

De igual manera se empleó el método estructural funcional para la construcción de la propuesta, la medición, el experimento (pre-experimentos), la técnica de distribución empírica de frecuencia, el análisis estadístico e inferencial, entre otros.

Para dar respuesta al objetivo que alude a la validación empírica, se definió una hipótesis de investigación, la cual expresa que:

Hi: Un sistema de entrenamiento combinado mejora el desarrollo de la fuerza explosiva de los miembros inferiores en los taekwondocas universitarios de la categoría sénior

Al estar complementada el desarrollo de la fuerza explosiva con la Fuerza máxima (FMax) se define una hipótesis estadística, para monitorear la significatividad de los cambios de la FMax en los planos musculares que intervienen directamente en las acciones técnicas ejecutadas con el tren inferior. Como resultado del análisis estructural funcional de las acciones se definió concentrar la prueba de FMax (1RM) en el Bíceps femoral, Gemelos y Cuádriceps, de manera que se declara la siguiente hipótesis de correlación:

$\mathrm{H}_{0}$ : Existe una correlación lineal ente los niveles de fuerza máxima de los planos musculares que intervienen en la ejecución de las acciones técnicas del tren inferior de los taekwondocas y la fuerza explosiva

$\mathrm{H}_{1}$ : no existe una correlación lineal ente los niveles de fuerza máxima de los planos musculares que intervienen en la ejecución de las acciones técnicas del tren inferior de los taekwondocas y la fuerza explosiva

Para comprobar la hipótesis estadística se realizó un análisis de las posibles relaciones entre las variables de Fuerza explosiva con los resultados de las pruebas de Fuerza máxima de Bíceps femoral, Gemelos y Cuádriceps, utilizando la prueba de correlación de Pearson.

El valor del índice de correlación varía en el intervalo [$1,1]$, indicando el signo el sentido de la relación:

- $\quad$ Si $r=1$, existe una correlación positiva perfecta. El índice indica una dependencia total entre las dos variables denominada relación directa: cuando una de ellas aumenta, la otra también lo hace en proporción constante.

- $\quad \operatorname{Si} 0<\mathrm{r}<1$, existe una correlación positiva.

- $\quad$ Si $r=0$, no existe relación lineal. Pero esto no necesariamente implica que las variables son independientes: pueden existir todavía relaciones no lineales entre las dos variables.

- $\quad \mathrm{Si}-1<\mathrm{r}<0$, existe una correlación negativa.

- $\quad \mathrm{Si} r=-1$, existe una correlación negativa perfecta. El índice indica una dependencia total entre las dos variables llamada relación inversa: cuando una de ellas aumenta, la otra disminuye en proporción constante.

La dinámica del pre-experimento contó con un primer momento donde se implementaron las pruebas declaradas 
para medir la fuerza máxima (1RM) y fuerza explosiva (Prueba de Salto vertical o Squat Jump: SJ), posteriormente se desarrolló el proceso de intervención con una previa preparación de los entrenadores para asegurar el despliega del sistema de entrenamiento combinado durante 16 semanas, vale clarificar que se profundizó en el manejo de las bases teóricas y metodológicas de la propuesta, manejo de las cargas (Volumen, Zonas de Intensidad y Densidad), empleo del sistema de ejercicio que ofrece la alternativa de solución como respaldo de la preparación, cambios estructurales y funcionales por zonas y etapas de la preparación, entre otros elementos de reveladora importancia. Se evaluó en un segundo momento (Post-test) las capacidades bajo las mismas condiciones y exigencia que en el primer momento.

Para el análisis estadístico e inferencial de los datos se realizó de forma previa un test de bondad de ajustes, en este caso fue el test de Koogorov-Smirnov (KS), cuyo objetivo consistió en determinar el comportamiento de la distribución de los datos, se identifica la normalidad de los datos y se procede a contrastar los resultados del pre y el post-test utilizando una prueba paramétrica: prueba $\mathrm{T}$ para diferencia de medias, la cual permitió identificar la significatividad de los cambios asumiendo como valor prefijado de $p=.05$.

\section{Presentación de la propuesta}

Los resultado de la fase diagnóstica permitieron diseñar la propuesta y atemperarla a las necesidades del estado actual del objeto de estudio, proceso en el cual se constataron altas limitaciones por parte de los entrenadores del equipo universitario de la categoría Sénior de la Universidad Central del Ecuador, en relación al manejo de las bases teóricas y metodológicas para la planificación del entrenamiento deportivo, selección y empleo de medios auxiliares y específicos para la consecución del desplazamiento de la fuerza y su conversión hacia la fuerza explosiva, dominio de la dinámica de la planificación según el carácter de los esfuerzos, manejo de los componentes de la carga y de las bases biológicas del ejercicio físico para el desarrollo de las base de la fuerza explosiva, en conclusión estos fueron los referentes que orientaron la delimitación de los elementos y componentes del Sistema de Entrenamiento diseñado como alternativa de solución.

Para la elaboración de la propuesta se consideró los criterios de Marcelo-Arnold \& Osorio (2003) al sostener que un sistema se define como un «Conjunto de elementos que guardan estrecha relación entre sí, que mantienen la es- tructura directa o indirectamente unidas de forma más o menos estable y cuyo comportamiento global persigue, normalmente un objetivo.» Estos pueden clasificarse según sus aperturas o intercambio con el medio en abiertos y cerrados, entre otros.

Según los autores el sistema como producto presenta diferentes propiedades formales, dentro de las que se encuentran: crecimiento, competencia, totalidad, sumatividad, segregación progresiva, orden jerárquico, complejidad, jerarquización y adaptabilidad.

Cabe destacar que el sistema, como resultado científico, responde a una necesidad de la práctica y se sustenta en determinada teoría, que en nuestro caso se apega a la teoría y metodología del Entrenamiento Deporte; en conclusión, la propuesta quedó estructuralmente con una entrada un proceso y una salida, estrechamente relacionadas, y cuenta con los siguientes elementos y componentes:

- Objetivo general de la propuesta

\section{- Entrada}

- Objetivo general

- Fundamentación teórica de la propuesta

- Sistema de principios que rigen el sistema

- Planificación gráfica del sistema de entrenamiento combinado

- Representación gráfica de los elementos estructurales y procesuales del sistema de ejercicio

\section{- Proceso}

1. Primer grupo de ejercicio: dirigidos a crear las condiciones morfológicas y funcionales

2. Segundo grupo de ejercicio: dirigidos a incrementar la fuerza máxima

3. Tercer grupo de ejercicio: dirigidos a desarrollar la fuerza explosiva

- Salida

- Sistema de evaluación

- Orientaciones metodológicas

Sistema de entrenamiento combinado para desarroIlar la fuerza explosiva de los miembros inferiores en los taekwondocas

Objetivo general: Optimizar el entrenamiento de la fuerza explosiva, con arreglo a la estructura y función de las acciones y el carácter de los esfuerzos

A continuación, se presenta la Planificación Gráfica del sistema de entrenamiento combinado (Figura 1)

El sistema de entrenamiento combinado posee internamente un sistema de ejercicios complementados entre sí y

\begin{tabular}{|c|c|c|c|c|c|c|c|c|c|c|c|c|c|c|c|c|c|c|}
\hline \multirow{3}{*}{\begin{tabular}{|l|} 
Periodos \\
Etapas \\
Semanas \\
\end{tabular}} & \multicolumn{14}{|c|}{ Periodo Preparatorio } & \multicolumn{4}{|c|}{ Periodo Competitivo } \\
\hline & \multicolumn{8}{|c|}{ Etapa de Preparación General } & \multicolumn{6}{|c|}{ Etapa Especial } & \multicolumn{2}{|c|}{$\begin{array}{l}\text { Etapa Pre- } \\
\text { Competitiva }\end{array}$} & \multicolumn{2}{|c|}{ Etapa Competitiva } \\
\hline & \multirow{2}{*}{\begin{tabular}{|c|}
1 \\
Introductorio \\
\end{tabular}} & \multirow{2}{*}{\multicolumn{2}{|c|}{\begin{tabular}{|c|c|}
2 & 3 \\
Básico Desarrollador \\
\end{tabular}}} & \multirow{2}{*}{\multicolumn{2}{|c|}{\begin{tabular}{|c|c|}
4 & 5 \\
Básico Estabilizador \\
\end{tabular}}} & 6 & 7 & 8 & 9 & 10 & 11 & 12 & 13 & 14 & & 16 & 17 & $18 / \mathrm{CF}$ \\
\hline Mesociclos & & & & & & \multicolumn{3}{|c|}{\begin{tabular}{|l|l|} 
Básico Desarrollador \\
\end{tabular}} & \multicolumn{3}{|c|}{ Básico Desarrollador } & \multicolumn{3}{|c|}{ Control Preparatorio } & \multicolumn{2}{|c|}{ Pre-Competitiva } & \multicolumn{2}{|c|}{ Competitiva } \\
\hline Microciclos & I & $\mathbf{0}$ & $\mathbf{0}$ & $\mathbf{0}$ & $\mathbf{R}$ & $\mathbf{O}$ & CH & $\mathbf{R}$ & $\mathbf{O}$ & $\mathbf{0}$ & $\mathbf{R}$ & $\mathbf{O}$ & CH & M & \begin{tabular}{l|l}
$\mathbf{A}$ & \\
\end{tabular} & $\mathbf{A}$ & \begin{tabular}{l|l}
$\mathbf{C}$ \\
\end{tabular} & $\mathbf{C}$ \\
\hline Proporción PG/PE & 4:1 & 4:1 & 4:1 & 4:1 & $3: 2$ & $3: 2$ & 4:1 & $3: 2$ & $2: 3$ & $1: 4$ & $2: 3$ & $2: 3$ & $1: 4$ & $1: 4$ & $1: 4$ & $1: 4$ & $1: 4$ & $1: 4$ \\
\hline Etapas & Test & AA & AA & Hipert & Hipert & Hipert & Hipert & Hipert & FMax & FMax & FMax & Potencia & FMax & Potencia & Potencia & Potencia & Potencia & \\
\hline V. \% 1RM & & $30-40 \%$ & $30-40 \%$ & $60-70 \%$ & $65-70 \%$ & $70-75 \%$ & $76-80 \%$ & $65-75 \%$ & $80-84 \%$ & $85-90 \%$ & $80-84 \%$ & $50-70 \%$ & $90-100 \%$ & $60-80 \%$ & $60-70 \%$ & $60-70 \%$ & $50-60 \%$ & \\
\hline Densidad & & 2 & 3 & 3 & 2 & 3 & 3 & 2 & 2 & 3 & 2 & 3 & 3 & 2 & 3 & 2 & 2 & \\
\hline Intensidad & & R2 & R2 & R2 & R2 & R3 & R3 & R3 & $\mathrm{R} 4$ & R4 & $\mathrm{R} 4$ & R5 & R5 & R5 & R5 & R5 & R5 & \\
\hline FCMax & & $60-65 \%$. & $60-70 \%$ & $65-70 \%$ & $65-70 \%$ & $70-75 \%$ & $70-75 \%$ & $75-80 \%$ & $80-84 \%$ & $80-85 \%$ & $85-90 \%$ & $90-100 \%$ & $90-100 \%$ & $90-100 \%$ & $90-100 \%$ & $90-100 \%$ & $90-100 \%$ & \\
\hline \multirow[t]{2}{*}{ Zona de I } & & \multicolumn{3}{|c|}{$\begin{array}{c}\text { Se Incrementa el ritmo metabólico. } \\
\text { Zona aerobia }\end{array}$} & \multicolumn{2}{|c|}{$\begin{array}{c}\text { Umbral Aerobio: punto } \\
\text { en el que las vias } \\
\text { energéticas anaeróbicas } \\
\text { comienzan a operar } \\
(65 \%-75 \%) \text {. }\end{array}$} & $\begin{array}{c}\text { Zona } \\
\text { aerobia }\end{array}$ & $\begin{array}{c}\text { Zona } \\
\text { Umbral } \\
\text { Aerobio } \\
\text { (Mixta) }\end{array}$ & $\begin{array}{c}\text { Zona } \\
\text { anaerobia }\end{array}$ & \begin{tabular}{|c|} 
Zona \\
anaerobia
\end{tabular} & $\left|\begin{array}{c}\text { Zona } \\
\text { Umbral } \\
\text { anaeróbico }\end{array}\right|$ & $\begin{array}{c}\text { Zona } \\
\text { anaerobia }\end{array}$ & $\begin{array}{c}\text { Zona } \\
\text { anaerobia }\end{array}$ & $\begin{array}{c}\text { Zona } \\
\text { anaerobia }\end{array}$ & \begin{tabular}{|c|} 
Zona \\
anaerobia
\end{tabular} & $\begin{array}{c}\text { Zona } \\
\text { anaerobia }\end{array}$ & \begin{tabular}{|c|} 
Zona \\
anaerobia
\end{tabular} & \\
\hline & & \multicolumn{4}{|c|}{ Intensidad Submedia } & $\begin{array}{l}\text { Intensidad } \\
\text { Media }\end{array}$ & \multicolumn{2}{|c|}{ Intensidad Media } & \multicolumn{2}{|c|}{ Intensidad Submáxima } & \begin{tabular}{|c|}
$\begin{array}{l}\text { Intensidad } \\
\text { Submáxima }\end{array}$ \\
\end{tabular} & $\begin{array}{l}\text { Intensidad } \\
\text { Máxima }\end{array}$ & $\begin{array}{l}\text { Intensidad } \\
\text { Máxima }\end{array}$ & $\begin{array}{c}\text { Intensidad } \\
\text { Máxima }\end{array}$ & $\begin{array}{c}\text { Intensidad } \\
\text { Máxima }\end{array}$ & $\begin{array}{c}\text { Intensidad } \\
\text { Máxima }\end{array}$ & $\begin{array}{c}\text { Intensidad } \\
\text { Máxima }\end{array}$ & \\
\hline
\end{tabular}

Figura 1. Planificación Gráfica del sistema de entrenamiento combinado

Abreviaturas: I, Microciclo introductorio; O, Microciclo ordinario; CH, Microciclo de Choque Intensivo, R, Microciclo de recuperación; M, Microciclo de modelaje; A, Microciclo de aproximación; C, Microciclo competitivo; AA, Adaptación anatómica; Hpert, Hipertrofia sarcoplasmática; FMax, Fuerza Máxima; FExp, Fuerza Explosiva 
responde a las especificidades de cada una de las etapas, los ejercicios generales y específicos que satisfacen las exigencias de las etapas y los objetivos plateados, estos estructuraron a partir de la contrastación de las exigencias del ejercicio competitivo y las deficiencias metodológicas en la conducción del proceso de entrenamiento.

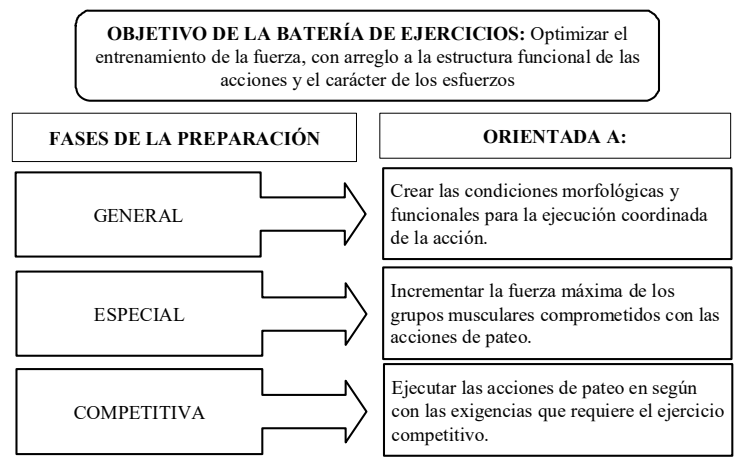

Figura 2. Representación esquemática de los objetivos del sistema de ejercicio por etapas.

\section{Primer grupo de ejercicios}

Objetivo específico: Crear las condiciones morfológicas y funcionales para la ejecución coordinada de la acción de pateo.

\section{Fundamentación}

Se trata de la integración sistémica de un conjunto de ejercicios para el tratamiento de los grupos musculares que se relacionan con la acción de patear, girar y desplazarse. Además, aunque las condiciones morfológicas se refieren al mejoramiento de las propiedades de los músculos, las funcionales se orientan a la optimización de los sistemas de abastecimiento energético que garantizan la resistencia para el trabajo y la calidad de la recuperación, lo que obliga a incluir otros ejercicios resistivos que conducirían a la formación de una gran red capilar que garantice el flujo de sustancias nutritivas, así como el $\mathrm{O}_{2}$ y la posibilidad de evacuación de $\mathrm{CO}_{2}$ creando de esa manera la posibilidad para mayor consumo de $\mathrm{O}_{2}$, a fin de enfrentar las exigencias de esfuerzos de mayor especificidad.

La aplicación de estos ejercicios se desarrollará predominantemente durante la preparación general, cuya duración es de siete (7) semanas, teniendo en cuenta que se partirá por la Adaptación Anatómica (AA) empleando ejercicios naturales durante tres (3) semanas de las siete (7) declaradas para esta etapa, para posteriormente entrar en el trabajo de hipertrofia muscular (4 semanas) que distingue lo efectos de las tareas de esta fase de preparación.

\begin{tabular}{|c|c|c|}
\hline No. Descripción de los medios & Dosificación & Aspectos procedimentales \\
\hline $1^{\circ}$. Carrera continua & 30 a $40 \mathrm{~min}$ & Los ejercicios que se relacionan \\
\hline $\begin{array}{l}2^{\circ} . \\
\text { cruzando las piernas }\end{array}$ & $3 \mathrm{~s} \times 3 \mathrm{r} \times 1 \mathrm{~d}$ & $\begin{array}{l}\text { tienen un carácter general con } \\
\text { respecto a la estructura de las }\end{array}$ \\
\hline $\begin{array}{l}3^{\circ} . \text { Elevación de talón con } \\
\text { mancuerna. }\end{array}$ & $4 \mathrm{~s} \times 8 \mathrm{r} \times 2 \mathrm{~d} 60 \%-70 \%$ & $\begin{array}{l}\text { acciones de pateo. Sin embargo, } \\
\text { la carrera continua y el efecto }\end{array}$ \\
\hline $4^{\circ}$. Extensión de talones sentado. & $4 \mathrm{~s} \times 8 \mathrm{r} \times 2 \mathrm{~d} 60 \%-70 \%$ & acumulado del resto de los \\
\hline $5^{\circ}$. Extensión de la pierna hacia atrás & $4 \mathrm{~s} \times 8 \mathrm{r} \times 2 \mathrm{~d} 60 \%-70 \%$ & ejercicios contribuye al \\
\hline $6^{\circ}$. Abductores con polea & $\begin{array}{l}4 \mathrm{~s} \times 8 \mathrm{r} \times 2 \mathrm{~d} \\
60 \%-70 \% \\
\end{array}$ & $\begin{array}{l}\text { incremento de la red capilar y al } \\
\text { desarrollo de la capacidad }\end{array}$ \\
\hline $7^{\circ}$. Curl de piernas parado. & $4 \mathrm{~s} \times 8 \mathrm{r} \times 2 \mathrm{~d} 60 \%-70 \%$ & ventilatoria, lo que permitirá \\
\hline $8^{\circ}$. Extensión de piernas sentado. & $4 \mathrm{~s} \times 8 \mathrm{r} \times 2 \mathrm{~d} 60 \%-70 \%$ & incrementar el consumo máximo \\
\hline $\begin{array}{l}9^{\circ} . \begin{array}{l}\text { Flexión lateral del tronco en } \\
\text { banco. }\end{array} \\
\end{array}$ & $3 \mathrm{~s} \times 15 \mathrm{r} \times 1 \mathrm{~d}$ & $\begin{array}{l}\text { de oxígeno, que es la base para } \\
\text { los esfuerzos anaeróbicos y al }\end{array}$ \\
\hline $10^{\circ}$. Elevación de rodillas en paralelas & $4 \mathrm{~s} \times 8 \mathrm{r} \times 2 \mathrm{~d} 60 \%-70 \%$ & mismo tiempo se fortalecen de \\
\hline $11^{\circ}$. Extensión del tronco acostado & $3 \mathrm{~s} \times 15 \mathrm{r} \times 1 \mathrm{~d}$ & $\begin{array}{l}\text { forma aislada aquellos músculos } \\
\text { que se comportan como } \\
\text { componentes de las cadenas } \\
\text { cinemáticas comprometidas con } \\
\text { las acciones. }\end{array}$ \\
\hline
\end{tabular}

Los métodos tendrán un carácter predominantemente extensivo. Es decir, con un nivel de intensidad moderado para trabajos de media y larga duración.

\section{Segundo grupo de ejercicios}

Objetivo específico: Incrementar la fuerza máxima de los grupos musculares comprometidos con las acciones técnicas ejecutadas con el tren inferior: pateos, giros, desplazamientos

\section{Fundamentación}

Si bien la dosificación de los ejercicios del grupo anterior resultaba moderada por su finalidad. Ahora, al proyectarse al incremento de la fuerza máxima, no solo aumenta el porciento del peso externo que se comporta como resistencia, sino que los ejercicios seleccionados integran una mayor cantidad de los músculos comprometidos con la cadena cinemática que determina la acción de patear, unido a ellos deben considerarse los ejercicios de coordinación para asegurar los procesos de transferencia que optimizan la calidad de la técnica de ejecución.

Los esfuerzos resistivos, dejan de ser moderado para convertirse en potencia aeróbica, a fin de garantizar una mayor velocidad de oxidación de los sustratos en las crestas mitocondriales, aprovechando el mejoramiento de las propiedades de las fibras musculares ya alcanzado y potenciando la posibilidad de resistir con éxito los esfuerzos específicos de la preparación, lo que sirve de soporte para tolerar en el futuro, el trabajo anaeróbico propio del ejercicio competitivo.

Este conjunto de ejercicios se aplicará fundamentalmente durante la preparación especial, la cual tendrá una duración de seis (6) semanas y una de sus principales finalidades es el incremento de la fuerza máxima, la cual durante las últimas dos (2) o tres semanas de esta etapa se orientará no solo al incremento de los índices de la fuerza, sino al de transferencia, entendida como el tránsito de un fortalecimiento general, a la potenciación y relación de los músculos que forman parte de las cadenas cinemáticas de las acciones específicas de pateo y compensación de sus esfuerzos, lo que se distingue por un aumento de la especificidad de las tareas de entrenamiento, a partir de la repetición en un régimen sub máximo de trabajo, propio de métodos entre intensivos de corta duración y extensivos de duración media.

\begin{tabular}{|c|c|c|c|}
\hline No. & $\begin{array}{l}\text { Descripción de los } \\
\text { medios }\end{array}$ & Dosificación & Aspectos procedimentales \\
\hline $1^{\circ}$. & $\begin{array}{l}\text { Carrera a máxima } \\
\text { velocidad. }\end{array}$ & 6 a 8 r x 90" d & \multirow{11}{*}{$\begin{array}{l}\text { Una vez lograda la base necesaria, } \\
\text { tanto desde la resistencia como } \\
\text { _desde la fuerza, se introducen los } \\
\text { _ejercicios que integran los grupos } \\
\text { musculares comprometidos con las } \\
\text { acciones de pateo y se incluyen } \\
\text { _otros vinculados a la rapidez, } \\
\text { teniendo en cuenta que esta se } \\
\text { identifica con el carácter de los } \\
\text { oesfuerzos. Nótese que la }\end{array}$} \\
\hline $2^{\circ}$. & Saltos laterales. & $3 \mathrm{~s} \times 8 \mathrm{r} \times 90 " \mathrm{~d}$ & \\
\hline $3^{\circ}$. & Saltos con giros. & $3 \mathrm{~s} \times 8 \mathrm{r} \times 90 " \mathrm{~d}$ & \\
\hline $4^{\circ}$. & Ejercicios acrobáticos. & $10 \mathrm{~min}$ & \\
\hline $5^{\circ}$ & $\begin{array}{l}\text { Peso muerto con piernas } \\
\text { semirrígidas. }\end{array}$ & $\begin{array}{l}\text { 4t x 1-6r x90" d } \\
80 \%-100 \%\end{array}$ & \\
\hline $6^{\circ}$. & $\begin{array}{l}\text { Extensión del tronco en } \\
\text { banco. }\end{array}$ & $4 \mathrm{t} \times 6 \mathrm{r} \times 90 " \mathrm{~d} 80 \%-100 \%$ & \\
\hline $7^{\circ}$. & Torsiones con bastón. & $4 \mathrm{t} \times 1-6 \mathrm{r} \times 90 " \mathrm{~d} 80 \%-100 \%$ & \\
\hline $8^{\circ}$. & Squat o sentadilla. & $4 \mathrm{t} \times 1$-6r x $90 " \mathrm{~d} 80 \%$ & \\
\hline $9^{\circ}$. & $\begin{array}{l}\text { Prensa de piernas } \\
\text { inclinada. }\end{array}$ & 4t x 1-6r x90" d $80 \%-100 \%$ & \\
\hline $10^{\circ}$ & $\begin{array}{l}\text { Elaciones del tronco en } \\
\text { suspensión en el banco. }\end{array}$ & $3 \mathrm{~s} \times 15 \mathrm{r} \times 1 \mathrm{~d}$ & \\
\hline $11^{\circ}$. & $\begin{array}{l}\text { Flexión lateral de tronco } \\
\text { con mancuerna. }\end{array}$ & $3 \mathrm{~s} \times 15 \mathrm{r} \times 1 \mathrm{~d}$ & \\
\hline
\end{tabular}

\section{Tercer grupo de ejercicios}

Objetivo específico: Desarrollar la fuerza explosiva en las acciones de pateo según las exigencias que requiere el ejer- 
cicio competitivo.

\section{Fundamentación}

El hecho de que el taekwondo es un deporte de esfuerzo variable a partir de acciones cuyas estructuras se caracterizan por ser acíclicas variadas, determina tanto los ejercicios como sus formas de organización en la estructura de la tarea de entrenamiento. Por otra parte, dado el carácter de los esfuerzos, se aprecia con mayor intervención la fuerza explosiva, que depende de la relación entre la fuerza y la rapidez, acciones en las que la coordinación se erige como condición indispensable, así como la intervención del sistema nervioso que comenzó a activarse desde la introducción de la fuerza máxima en la fase anterior.

La capacidad lactácida permite soportar el tiempo de duración del combate o la demostración de las pumses y la potencia lactácida distingue a las acciones explosivas que fueron observadas durante la caracterización del ejercicio competitivo y las demostraciones de rompimiento.

Por último, el siguiente grupo de ejercicios, el cual tiene la mayor especificidad, se aplicará en la fase competitiva de la preparación, que tiene una duración de cuatro semanas, su finalidad máxima es la transferencia de los índices de fuerza máxima a los valores de la fuerza explosiva, que se distinguen por la potencia en la ejecución de acciones explosivas, utilizando como medios específicos aquellos subsistemas de movimientos que forman parte de la estructura de las acciones de pateo. Los métodos a aplicar responden fundamentalmente a un régimen intensivo para trabajos de corta o muy corta duración.

\begin{tabular}{|c|c|c|}
\hline No. Descripción & Dosificación & Aspectos procedimentales \\
\hline 1. Ejercicios acrobáticos. & $10 \mathrm{~min}$ & Este grupo de ejercicios tiene \\
\hline 2. Saltos laterales y pateo. & $4 \mathrm{~s} \times 8 \mathrm{r} 90 " \mathrm{~d}$ & mayor especificidad y pretende \\
\hline 3. Saltos con giros y pateo. & $4 \mathrm{~s} \times 8 \mathrm{r} 90 " \mathrm{~d}$ & mantener los niveles de fuerza \\
\hline 4. Extensión del tronco en banco. & $3 \mathrm{~s} \times 15 \mathrm{r} \times 1 \mathrm{~d}$ & alcanzados, teniendo en cuenta que \\
\hline $\begin{array}{l}\text { 5. Elevación simultanea de } \\
\text { piernas y tronco en el suelo. }\end{array}$ & $4 \mathrm{~s} \times 8 \mathrm{r} 90$ " d & $\begin{array}{l}\text { el entrenamiento de la rapidez } \\
\text { ayuda a mantener los indices de }\end{array}$ \\
\hline $\begin{array}{l}\text { Flexión lateral de tronco con } \\
\text { mancuerna. }\end{array}$ & $\begin{array}{l}\text { 3s x 6r 90"d } \\
30 \%-80 \% \\
\end{array}$ & $\begin{array}{l}\text { fuerza. Sin embargo, se mantienen } \\
\text { los ejercicios propiamente de }\end{array}$ \\
\hline 㧫. Squat o sentadilla. & $3 \mathrm{~s} \times 6 \mathrm{r} 90 " \mathrm{~d} 30 \%-80 \%$ & fuerza, con un porciento \\
\hline$\overline{8^{\circ}}$. Prensa de piernas inclinada. & $3 \mathrm{~s} \times 6 \mathrm{r} 90 " \mathrm{~d} 30 \%-80 \%$ & relativamente bajo, de modo que se \\
\hline $\begin{aligned} & \text { Serie combinada de: } \\
&- \text { Saltos al frente } \\
&- \text { Prensa de piernas } \\
& \text { inclinada. } \\
& 9^{\circ} . \text { Hiperextensión. } \\
&- \text { Saltos laterales } \\
&- \text { Elevación simultanea de } \\
& \text { piernas y tronco en el } \\
& \text { suelo. } \\
&\end{aligned}$ & $4 \mathrm{~s} \times 20 " \times 2 \mathrm{mp}$ & $\begin{array}{l}\text { puedan realizar las acciones a la } \\
\text { máxima velocidad que es condición } \\
\text { para conseguir la fuerza explosiva } \\
\text { que identifica a las acciones de } \\
\text { taekwondo. Se recomienda alternar } \\
\text { los esfuerzos excéntricos con los } \\
\text { concéntricos. }\end{array}$ \\
\hline
\end{tabular}

\section{Sistema de evaluación}

La prueba de fuerza máxima se realizará de forma individual, asistida y monitoreada por los entrenadores:

1. Asignar inicialmente el peso dominio del atleta, registrado por el profesor

2. Se inicia la prueba con el $80 \%$ del RM Individual del deportista (última prueba)

3. Se incrementa el peso de forma progresiva y creciente, hasta que el atleta considere que se encuentra al límite de sus esfuerzos

\section{Orientaciones metodológicas generales}

La relación sistémica que tiene lugar entre los ejercicios dentro de cada grupo es de carácter progresivo, pero ese mismo modo de relación se manifiesta entre los grupos de ejercicios que componen el sistema, lo que evidencia que el diseño y la selección de los ejercicios y grupos de ejercicios se fundamenta en el principio de sistematización y profundidad del contenido. No obstante, se respeta la flexibilidad como propiedad del sistema y su adaptabilidad al contexto con el que se relaciona, ya que no solo se puede, sino que se deben alternar e intercalar en el proceso con arreglo a los objetivos de cada una las sesiones de trabajo.

El criterio de progresión de la dificultad y complejidad con vistas al aumento de las cargas va desde las flexiones frontales a las laterales y de estas a las torsiones o rotaciones internas y externas.

Por último, se recomienda que después de las sesiones de trabajo, se apliquen ejercicios de estiramientos para estimular la propiedad esponjosa de los músculos implicados y ejercicios de esfuerzos compensatorios para acelerar el proceso de recuperación por una parte y por la otra, para lograr un desarrollo equilibrado de la fuerza.

La alternancia entre ejercicios excéntricos y concéntricos durante el entrenamiento, estimuló las propiedades elásticas de los músculos y en consecuencia contribuyó a la optimización de los esfuerzos explosivos.

\section{Resultados}

Para proceder con la contrastación se inició con un análisis estadístico para conocer si los datos de las variables provienen de una distribución normal, al ser $p$ e» .05 en todas las variables se puede concluir provienen de una distribución normal, tal y como se muestra en la tabla (4), lo se justifica la utilización de la prueba paramétrica de diferencias de media para muestras relacionadas.

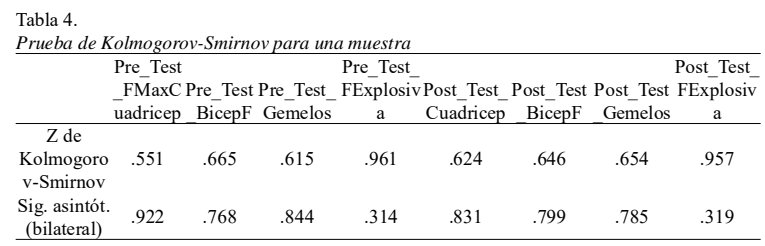

$\mathrm{Al}$ analizar los datos aportados por los entrenadores del estado de la fuerza máxima a inicio de la etapa de preparación física general (Pre-test), pudimos apreciar que los valores en la Fuerza máxima en el cuádriceps, en el sexo femenino, se mostraron entre 39 y $79 \mathrm{~kg}$ y en el sexo masculino entre 48 y $110 \mathrm{~kg}$, de forma general los resultados en el equipo estuvieron en un rango de $39 \mathrm{~kg}$ y $110 \mathrm{~kg}$, con una media $69 \mathrm{~kg}$, en este momento los datos se mantuvieron alrededor de la media con una desviación de 20.01. En el post-test los resultados en la prueba se incrementaron tanto en el sexo masculino como en el femenino, sin embargo, de forma general los datos oscilaron entre $49 \mathrm{~kg}$ y $123 \mathrm{~kg}$, con una media de 76.5 $\mathrm{kg}$ superior al pre-test en 7.35 , con una desviación típica mayor que en el pre-test con 21.55 , ya que los valores se mostraron más dispersos que en el primer momento.

En relación a la prueba de fuerza máxima de Bíceps Femoral podemos indicar que los registros en el primer momento estuvieron en un rango de 18 y $84 \mathrm{~kg}$, con una media de 44.30, y una desviación típica de 21.18 , sin embargo en el sexo femenino los valores oscilaron entre 18 y $37 \mathrm{~kg}$ y en el sexo 
masculino entre 37 y $84 \mathrm{~kg}$, sin embargo al analizar el posttest constatamos un incremento de la media en 4.95, de forma general en el equipo, ya que en el post-test los rangos se mostraron entre 22 y $87 \mathrm{~kg}$, con una media de 49.25 superior en $4.95 \mathrm{~kg}$ y una desviación típica inferior al pre-test (20.99) de 3.18 , lo que devela una diferencia significativa entre ambos momentos.

En relación la prueba de fuerza máxima en los gemelos, en el primer momento, se pudo notar que los valores de la prueba se mostraron entre 108 y $278 \mathrm{~kg}$, con una media de $183 \mathrm{~kg}$ y una desviación típica 56.74, de forma particular en el sexo femenino, durante el post-test, se datos mostraron entre 108 y $200 \mathrm{~kg}$ y en el sexo masculino se fijaron en un rango de 142 y $278 \mathrm{~kg}$. En el segundo momento los resultados se desplazaron en positivo con cambios significativos al incrementarse la media en $9.7 \mathrm{~kg}(193.25 \mathrm{~kg})$, el rango osciló entre 114 y 289 $\mathrm{kg}$, con una desviación típica mayor que el pre-test (58.42), lo que indica que en el segundo momento los datos se mostraron más dispersos.

\begin{tabular}{|c|c|c|c|c|c|}
\hline & & Media & $\mathrm{N}$ & Desviación típ. & Error típ. de la media \\
\hline \multirow[t]{2}{*}{ Par 1} & Pre_Test_FMaxCuadricep & 69.0000 & 20 & 20.01578 & 4.47567 \\
\hline & Post Test Cuadricep & 76.3500 & 20 & 21.55354 & 4.81952 \\
\hline \multirow[t]{2}{*}{ Par 2} & Pre_Test_BicepF & 44.3000 & 20 & 21.18117 & 4.73625 \\
\hline & Post_Test_BicepF & 49.2500 & 20 & 20.99593 & 4.69483 \\
\hline \multirow[t]{2}{*}{ Par 3} & Pre_Test_Gemelos & 183.5500 & 20 & 56.74270 & 12.68805 \\
\hline & Post_Test_Gemelos & 193.2500 & 20 & 58.42393 & 13.06399 \\
\hline \multirow[t]{2}{*}{ Par 4} & Pre_-Test_FExplosiva & 30.5300 & 20 & 5.40595 & 1.20881 \\
\hline & Post Test FExplosiva & 33.0150 & 20 & 5.95892 & 1.33245 \\
\hline
\end{tabular}

momento con 5.9, lo que indica que los datos se encontraron más disperso alrededor de la media.

A través del análisis estadístico e inferencial de las tres pruebas de Fuerza Máxima se pudo constatar que se produjeron cambios significativos entre el pre y el post test al ser el valor de $p=.000$ en todos los casos, tal y como muestra la tabla (6).

El análisis de la relación entre la fuerza explosiva y la fuerza máxima de cada plano muscular implicado, arrojó una correlación lineal directa con las tres pruebas de FMax, variando el coeficiente de correlación «r» desde .651 (Fuerza máxima en cuádriceps) hasta .849 (Fuerza máxima bíceps femoral) (tabla 7). En los tres casos se puede observar que el coeficiente de correlación es altos, además al ser el coeficiente positivo, la correlación es positiva, lo expuesto se observa en los gráficos $(4,5,6)$, donde la pendiente de la recta de tendencia es positiva.

Tabla 7.

Coeficientes de correlación en Pre-test

\begin{tabular}{lcc}
\hline \multicolumn{2}{c}{$0<\mathrm{r}<1$, existe una correlación positiva } \\
\cline { 2 - 3 } $\begin{array}{c}\text { Correlación entre fuerzas máximas } \mathbf{y} \\
\text { explosivas }\end{array}$ & \multicolumn{2}{c}{ Fuerza explosiva } \\
\hline Fuerza máxima en cuádriceps & 0,651 & Sig Bilateral \\
\hline Fuerza máxima bíceps femoral & 0,849 & 0,002 \\
\hline Fuerza máxima en gemelos & 0,805 & 0,000 \\
\hline
\end{tabular}

Estadísticamente se pudo comprobar la hipótesis plateada, por tanto se verifica $\mathrm{H}_{0}$ y rechazar $\mathrm{H}_{1}$ indicando que

\section{Análisis estadístico}

En relación a la Fuerza explosiva, se pudo aprecia que en el primer momento los datos del equipo oscilaron entre 22 y $38.9 \mathrm{~cm}$, con una media de $30.53 \mathrm{~cm}$ y una desviación típica de 5.4; los valores en el sexo femenino oscilaron entre 22 y $27 \mathrm{~cm}$, con una media de $24.57 \mathrm{~cm}$, y en el sexo masculino los registros estuvieron en un rango de 30.9 a $39.9 \mathrm{~cm}$ con una media de $34.5 \mathrm{~cm}$. Dichos valores se incrementaron en el segundo momento de manera general, de esta forma los datos consignados registraron un mínimo de 23.9 cm y un valor máximo de 40.3 $\mathrm{cm}$, con una media de $33.01 \mathrm{~cm}$, incrementándose en $2.48 \mathrm{~cm}$ del pre al post tratamiento, registrando una desviación típica superior al primer

\begin{tabular}{|c|c|c|c|c|c|c|}
\hline & & & & \multirow{3}{*}{$\begin{array}{c}\text { Sig. } \\
\text { (bilateral }\end{array}$} \\
\hline & \multirow[b]{2}{*}{ Media } & \multirow[t]{2}{*}{$\begin{array}{l}\text { Desviación } \\
\text { típ. }\end{array}$} & \multicolumn{2}{|c|}{$\begin{array}{c}95 \% \text { Intervalo de } \\
\text { confianza para la } \\
\text { diferencia }\end{array}$} & \multirow[b]{2}{*}{$\mathrm{t}$} & \\
\hline & & & Superior & Inferior & & \\
\hline $\begin{array}{c}\text { Par } 1 \text { Pre_Test_FMaxCuadricep - } \\
\text { Post_Test_Cuadricep }\end{array}$ & -7.35 & 3.18343 & -8.83989 & -5.86011 & -10.325 & .000 \\
\hline $\begin{array}{c}\text { Par } 2 \text { Pre_Test_BicepF - } \\
\text { Post_Test_BicepF }\end{array}$ & -4.95 & 2.23548 & -5.99624 & -3.90376 & -9.903 & .000 \\
\hline $\begin{array}{l}\text { Par } 3 \text { Pre_Test_Gemelos - } \\
\text { Post_Test_Gemelos }\end{array}$ & -9.70 & 4.24388 & -1.68620 & -7.71380 & -10.222 & .000 \\
\hline $\begin{array}{r}\text { Par } 4 \text { Pre_Test_Fexplosiva - } \\
\text { Post Test_FExplosiva }\end{array}$ & -2.48 & 1.36739 & -3.12496 & -1.84504 & -8.127 & ,000 \\
\hline
\end{tabular}

existe una correlación positiva entre ambas variables, lo que justifica que el incremento de los niveles de la FMax desplace los niveles de Fuerza Explosiva en cada sujeto.

El análisis de los resultados indica que la variable independiente (sistema de entrenamiento combinado) mejoró la fuerza explosiva en cada sujeto, se comprueba en la contrastación del pre-test con post-test, que existen diferencia significativa $(p=.000)$. Al ser $p$ menor que el valor prefijado (.05), se acepta la hipótesis de investigación $\left(\mathrm{H}_{\mathrm{i}}\right)$ y se rechaza la hipótesis nula $\left(\mathrm{H}_{0}\right)$, lo que permite platear que el sistema de entrenamiento combinado mejoró el desarrollo de 


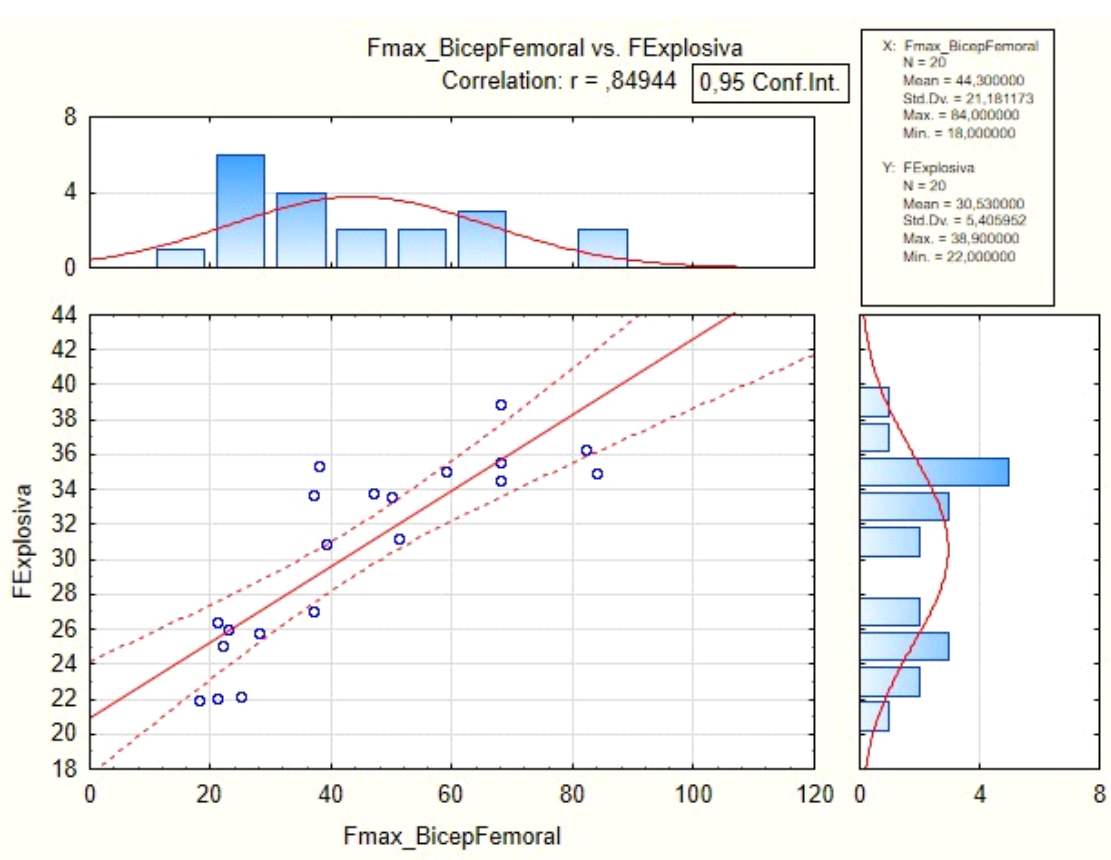

Figura 5. Cálculo del coeficiente de correlación de Pearson entre la fuerza explosiva y la fuerza máxima de Bíceps Femoral

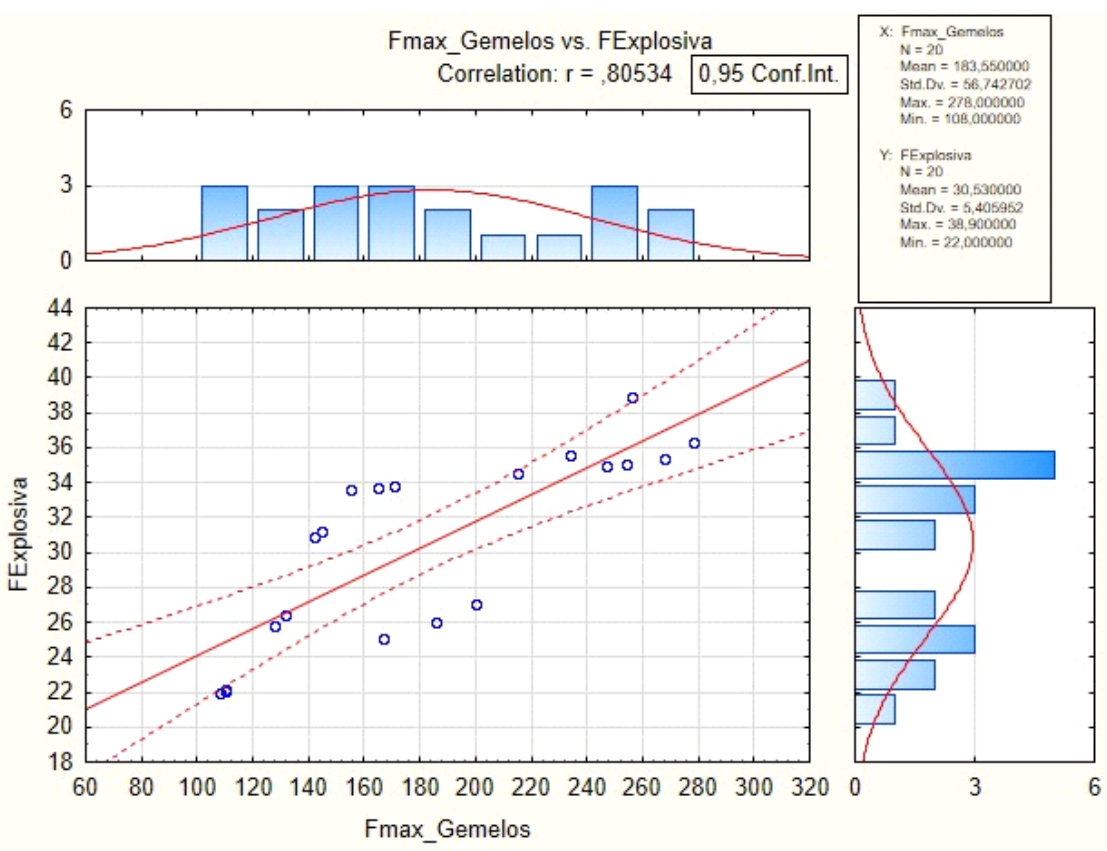

Figura 6. Cálculo del coeficiente de correlación de Pearson entre la fuerza explosiva y la fuerza máxima de Gemelo

la fuerza explosiva del tren inferior en los taekwondocas universitarios de la categoría sénior.

\section{Discusión de los resultados}

El presente estudio devela amplias dificultades en relación a la inadecuada planificación del desarrollo de la fuerza explosiva en los Taekwondocas de la Universidad Central del Ecuador, lo que coincide con los aportes de Ruales (2010), cuya investigación concuerda con las limitaciones planteadas sobre el objeto de estudio, el autor demuestra la viabilidad de un plan de entrenamiento para el desarrollo de la fuerza explosiva y su incidencia en el rendimiento físico del equipo de taekwondo de la Universidad de las Fuerzas Armadas del Ecuador.

Después de 16 semanas de entrenamiento con los atletas universitarios, se logró desplazar significativamente la fuerza máxima y la fuerza explosiva de los miembros inferiores, manifestándose una correlación lineal positiva entre ambas capacidades, se demostró que existe una correlación fuerte $(r<1)$ entre la fuerza máxima de los planos musculares que intervienen en la ejecución de las acciones técnicas de pateos, giros y desplazamientos de los taekwondocas y la fuerza explosiva, lo cual se mejoró como resultado de la intervención.

Los datos aportados en este estudio contrastan con los que exponen GarcíaAsencio, et al (2016) los cuales comprueban que un sistema de entrenamiento combinado centrado en el desarrollo de la fuerza mejora la capacidad de salto de los atletas en 16 semanas, de igual manera Sánchez-Sixto \& Floría (2017), presenta similares resultados, al respecto demuestra que un entrenamiento combinado de fuerza y pliometría es efectivo para conseguir mejorar en salto en jugadoras de baloncesto con lo cual coincide (PerezGomez \& Calbet, 2013),

Se demuestra que el test de salto vertical Squat jump (SJ), utilizando una plataforma de contacto digital, constituye una herramienta factible para determinar con precisión el desplazamiento de los niveles de fuerza explosiva de los miembros inferiores (Nikolaidis et al, 2015; Ojeda-Aravena et al., 2021) los valores permitieron develar una diferencia significativa entre el pre y el post tratamiento, lo que demostró la factibilidad de la alternativa de solución en el mejoramiento de la variable, resultados que contrastan con otros estudios analizados por Cardozo \& Moreno-Jiménez (2018).

Se demostró que un sistema de entrenamiento combinado con medios específicos para su desarrollo permite potenciar la fuerza explosiva como capacidad determinantes en la ejecución de las técnicas de pateos, giros y desplazamientos y que esta se encuentra altamente asociada a la Fuerza máxi- 
ma, lo que impone su desarrollo en la lógica metodológica de la planificación, resultados que concuerdan con otros estudios de espaecial relevancia, con similar metodología donde coinciden los resultados planteados, se justifica el valor de los procedimiento declarados y similares resultados para el desarrollo de la fuerza explosiva (Myong-Won, Hyun-Chul, Jong-Kook \& Hyun-Bae, 2015; Torrealba-Paéz et al., 2015; León et al., 2017; Monks et al., 2017).

\section{Conclusiones}

El diagnóstico permitió identificar la relación entre las palancas óseas y los grupos musculares que intervienen directamente en el pateo, en concordancia la evaluación de la fuerza máxima, se centró en el cuádriceps, bíceps femoral y gemelos.

Como resultado de la fase diagnóstica se pudo identificar amplios vacíos teóricos y metodológicos para el desarrollo de la fuerza explosiva, dificultades en el manejo de los sistema de planificación y de la base biológica para su concepción, deficiencias en la lógica metodológica para la gestión de la capacidad, y la selección de los medios específicos para su desarrollo en la dinámica de la preparación, así como de los criterios de dosificación.

Los resultados del diagnóstico sirvieron de base para la elaboración de un sistema de entrenamiento orientado al mejoramiento de la fuerza explosiva de los taekwondocas de la Categoría Sénior del Club de la Universidad Central del Ecuador, el cual fue validad en la práctica, donde se demostró su factibilidad en el mejoramiento de la variable relevante, este se caracterizó por su progresividad, flexibilidad y especificidad a partir de los elementos y componentes que lo conforman.

La evaluación de la fuerza máxima (1RM) y de la fuerza explosiva mediante un salto vertical Squat jump (SJ), permitieron comprobar la hipótesis estadística y la hipótesis de investigación plateada en el estudio, por lo que se demostró que existe una relación significativa entre el incremento de los niveles de fuerza máxima de los planos musculares que intervienen en la ejecución de las acciones técnicas ejecutadas con los miembros inferiores y el desplazamiento de los niveles de fuerza explosiva como resultado de la intervención de un sistema de entrenamiento combinado, por tanto se concluye que el sistema de entrenamiento mejoró el desarrollo de la fuerza explosiva de los miembros inferiores en los taekwondocas universitarios de la categoría sénior de la Universidad Central del Ecuador.

\section{Referencias}

Álvarez-Bedolla,A. (2003a). Organización eficiente y racional de los contenidos de preparación física en el Taekwondo de alta competición. Lecturas: Educación física y deportes. Revista Digital. Buenos Aires, 9(64). Recuperado de: http:// www.efdeportes.com/efd64/tkd.htm

Álvarez-Bedolla,A. (2003b). Selección de los contenidos para el desarrollo óptimo de la preparación física en competidores de Taekwondo. Lecturas: Educación física y deportes. Revista Digital - Buenos Aires, 8(58). Recuperado de: https:// docplayer.es/80846397-Seleccion-de-los-contenidos-para-el- desarrollo-optimo-de-la-preparacion-fisica-en-competidoresde-taekwondo.html

Bridge, C.A., Ferreira, J., Chaabene, H., Pieter, W., \& Franchini, E. (2014). Perfiles físicos y fisiológicos de atletas de taekwondo. Sports Med, 44(6), 713-733.

Bompa, T. O. (1996). Periodización del entrenamiento deportivo. Editorial: PAIDOTRIBO. España, 234.

Briñones, F. A., Camejo, R. C. E., \& Rosales, C. A. (2017). El entrenamiento de la potencia en el taekwondo. Lecturas: Educación física y deportes. Revista Digital. Buenos Aires, 21(226), Marzo. Recuperado de: http://www.efdeportes.com/ efd226/el-entrenamiento-de-la-potencia-en-eltaekwondo.htm

Bosco, C. (1994). La valoración de la fuerza con el test de Bosco. Colección Deportey Entrenamiento. Ed. Paidotribo. Barcelona.

Carazo, V. P. (2013). Respuestas y adaptaciones fisiológicas al entrenamiento de Taekwondo: Una revisión sistemática. Revista de Ciencias del ejercicio y la salud. Pensar en Movimiento. 11(2), 1-19.

Cardozo, L.A., \& Moreno-Jiménez, J. (2018). Valoración de la Fuerza Explosiva en Deportistas de Taekwondo: Una Revisión Sistemática. Kronos. 17 (1). Recuperado de: https:/gse.com/valoracion-de-la-fuerza-explosiva-en-deportistas-detaekwondo-una-revision-sistematica-2430-say5b4e14fcec173

Cardozo,L.A., Vera-Rivera,D.A., Conde-Cabezas, O.A., \&Yánez, C. A. (2017). Aspectos fisiológicos de deportistas elite de taekwondo: una revisión narrativa. Revista Española de Educación Física y Deportes, 418, 35-46

Celina, H., \& Campo, A. (2005). Aproximación al uso del coeficiente alfa de Cronbach. Revista colombiana de psiquiatría, (4), 572-580.

Cometti, G. (2002). El entrenamiento de la velocidad. Barcelona. Editorial Paidotribo

Crespo, B. T. (2007). Respuestas a 16 preguntas sobre el empleo de expertos en la investigación pedagógica. Editorial San Marcos. Lima. Perú, 235.

García-Orea, G., Heredia, E. J.,Aguilera, C. J.,Arenas-Dalla, C., \& Pérez-Caballero, C. (2017). Dispositivos para la Medición de la Velocidad de Ejecución en el Entrenamiento de la Fuerza: ¿Todos Valen para lo Mismo?, Journal International Journal of Physical Exercise and Health Science for Trainers, 1(2).

García-Asencio, C., Sánchez-Moreno, M., \& González-Badillo, J. J. (2016). Entrenamiento combinado de fuerza y ejercicios de saltos, efectos sobre el rendimiento en el salto vertical en un grupo de alto nivel de jugadores de voleibol durante una temporada completa de competición. Retos, 29, 140-143

González, C. (2011). Caracterización técnico-táctica de la competición de combate de alto nivel en Taekwondo. Efectividad de las acciones tácticas. Barcelona: Universidad de Barcelona.

González, C., Iglesias, X., Mirallas, J., \& Esparza, G. (2011). Sistematización de la acción táctica. Apunts. Educación Física y Deportes, 56-67.

González-Badillo, J. J., \& Gorostiaga, E. (1995). Fundamentos del entrenamiento de la fuerza. Barcelona: Inde, 53-63.

González-Badillo, J. J., \& Izquierdo-Redín, M. (2006). La carga de entrenamiento y el rendimiento en fuerza y potencia muscular. Encuentro sobre Alto Rendimiento Deportivo. Se- 
cretaria General para el Deporte. Instituto Andaluz del Deporte(1-20). Recuperado de: http://www.munideporte.com/ i magenes/d o c u mentacion / ficheros / 20060515093151badillo2.pdf

Guillén,P.,Ale,Y., \& Coral,A.E.G.(2017).Alternativametodológica con un enfoque técnico táctico para el proceso de enseñanza aprendizaje del taekwondo. Lecturas: Educación física y deportes. Revista Digital. Buenos Aires. 21(225). Recuperado de: http://www.efdeportes.com/efd225/alternativametodologica-del-taekwondo.htm

James, L. P., Haff, G. G., Kelly. V., \& Beckman. E. (2016). Haciauna determinación de las características fisiológicas que distinguen a los atletas exitosos de artes marciales mixtas: una revisión sistemática de la literatura deportiva de combate. Sports Med.46(10): 1525-51.

Kazemi, M., Waalen, J., Morgan, C., \& White,A. R. (2006). Un perfil de los competidores olímpicos de taekwondo. J Sports SciMed, 5, 114-121.

Kim, H., Stebbins, C., Chai, J., \& Song, J. (2011). Entrenamiento de Taekwondo y estado físico en adolescentes femeninas. $J$ Sports Sci, 29(2), 133-138.

Kim, H., Jung, H., Song, J., Chai, J., \& Lee, E. (2015). Un estudio de seguimiento sobre el físico, la composición corporal, la aptitud física y la fuerza isocinética de las atletas universitarias de Taekwondo. JExerc Rehabil, 11(1), 57-64.

Méndez, V. (2018). Acciones para incrementar la fuerza explosiva en el taekwondo de iniciación en la Universidad Técnica del Norte. Tesis en opción al título de Licenciado en Ciencias de la Actividad Física, Deportes y Recreación, Repositorio de la Universidad de Fuerzas Armadas ESPE. Recuperado de: http://repositorio.espe.edu.ec/handle/21000/ 14381

Marcelo-Arnold,A., \& Osorio, F. (2003). Introducción a los conceptos básicos de la teoría general de los sistemas. Facultad de Ciencias Sociales. Universidad Católica de Santiago de Chile. Recuperado de: http:// rehue.csociales.uchile.cl/ publicaciones/mosbic.htm

Martínez, H. L. E., Pegueros, P.A., Ortiz, A. A., del Villar, M.A., Flores, H.V., \& Pineda, V.C. (2014). Valoración isocinética de la fuerza y balance muscular del aparato extensor y flexor de la rodilla en taekwondo. Gaceta Médica de México. (3), 150272.

Monks, L., Seo, M., Kim, H., Jung, H., \& Song, J. (2017). Entrenamiento de intervalos de alta intensidad y rendimiento deportivo en atletas de Taekwondo. J Sports Med Phys Fitness. 57(10), 1252-1260.

Morales, C. Y. (2014a). La gestión del aprendizaje y el desempeño técnico-táctico del taekwondista escolar. Lecturas: Educación física y deportes. Revista Digital. Buenos Aires. 19(192). Recuperado de: http://www.efdeportes.com/ efd192/desempeno-tecnico-tactico-del-taekwondista.htm

Morales, C. Y.(2014b). Método reflexivo para la preparación técnico-táctica del taekwondista escolar. Lecturas: Educación física y deportes. Revista Digital. Buenos Aires, 19(195). Recuperado desde: http://www.efdeportes.com/efd195/metodoreflexivo-del-taekwondista.htm

Morales, C.Y.(2015). Sistema didáctico para la actuación competente del taekwondista escolar desde la preparación técnico-táctica. Editorial Universitaria Edeunif. Recuperado de: http://www.dspace.uce.edu.ec/bitstream/25000/20972/3/
T-UCE-0016-CUF-001-P.pdf

Myong-Won, S., Hyun-Chul, J., Jong-Kook, S., \& Hyun-Bae, K. (2015). Efecto de 8 semanas de entrenamiento de pretemporada sobre la composición corporal, el estado físico, la capacidad anaeróbica y la fuerza muscular isocinética en atletas universitarios masculinos y femeninos de taekwondo. J Exerc Rehabil11(2): 101-107.

Nikolaidis, P., Chtourou, H., Torres-Luque, G, Tasiopoulos, J., Heller, E., \& Padulo, J. (2015). Efecto de un período de preparación de seis semanas sobre las respuestas fisiológicas agudas a un combate simulado en jóvenes atletas de taekwondo de nivel nacional. Pubmed, 47: 115-25.

Norjali-Wazir, M., Van-Hiel, M., Mostaert, M., Deconinck, F., Pion, J., \& Lenoir, M. (2019). Identificación de características de rendimiento de élite en una pequeña muestra de atletas de taekwondo.Pubmed. 14(5): 10-1371.

León, Q. X., Ortiz, B. N., Lara, R. J., Marcillo, N. J., \& Guerrero, G H. (2017). Estrategia para potenciar la fuerza explosiva en el taekwondo escolar masculino. Lecturas: Educación física y deportes. Revista Digital. Buenos Aires. 22(232). Recuperado de: https://www.efdeportes.com/efd232/estrategia-parafuerza-explosiva-en-el-taekwondo.htm

Ojeda-Aravena,A.P.,Azócar-Gallardo, J., Hérnandez-Mosqueira, C., \& Herrera-Valenzuela, T. (2021). Relación entre la prueba de agilidad específica en taekwondo (tsat), la fuerza explosiva y la velocidad líneal en 5-m atletas de taekwondo de ambos sexos. Retos, 39, 84-89.

Perez-Gomez, J., \& Calbet, J. (2013). Training methods to improve vertical jump performance. The Journal of Sports Medicine and Physical Fitness, 53(4), 339-357

Prieto, G P., Sagat, P., Brahim, B. M., \& Sedlacek, J. (2020). Análisis de la veracidad de determinadas creencias asociadas habitualmente al entrenamiento de fuerza. Una revisión narrativa. Retos, 38, XXX-XXX

Ruales, H. (2010). Análisis de la fuerza explosiva en el rendimiento físico del equipo de taekwondo de la ESPE. Propuesta alternativa. Tesis en opción al título de Licenciado en Ciencias de la Actividad Física, Deportes y Recreación, Repositorio de la Universidad de Fuerzas Armadas ESPE. Recuperado de: http://repositorio.espe.edu.ec/handle/21000/ 1009

Sánchez-Sixto,A., \& Floría, P. (2017). Efecto del entrenamiento combinado de fuerza y pliometría en variables biomecánicas del salto vertical en jugadoras de baloncesto. Retos, 31, 114117.

Sihyun, Y., Sang-Kyoon, P., Sukhoon, Y., Ji, S. L., Sung, L., \& Jiseon, R. (2018). Comparación del entrenamiento propioceptivo y el entrenamiento de fuerza muscular para mejorar la capacidad de equilibrio de los atletas de Taekwondo Poomsae: ensayos controlados aleatorios. J Sports Sci Med, 17(3):445-454.

Torrealba-Paéz, M. D., Torrealba-Paéz, M. E., Bolivar-Monaterio, H., Pacífico-Casadiego, J., \& Núñez-Rodríguez, M. (2015). Metodología para mejorar la fuerza explosiva con pesas en la ejecución de los Poomsae de los atletas de taekwondo junior del Estado Cojedes. Lecturas: Educación física y deportes. Revista Digital. Buenos Aires. 19(200). Recuperado de: https:/ /www.efdeportes.com/efd200/mejorar-la-fuerza-explosivacon-pesas-en-taekwondo.htm

Zatsiorsky, V. M. (1989). Metrología deportiva. Editorial Pueblo y Educación. Ciudad de la Habana. Cuba. 